BNL-113903-2017-JA

\title{
Structural Evolution of Sub-10 nm Octahedral Platinum-Nickel Bimetallic Nanocrystals
}

\author{
Qiaowan Chang, Yuan Xu, Zhiyuan Duan, Fei Xiao, Fang Fu, \\ Youngmin Hong, Jeonghyeon Kim, Sang-Il Choi, Dong Su, Minhua Shao
}

Accepted by the Nano Letters

May 2017

Center for Functional Nanomaterials

Brookhaven National Laboratory

\author{
U.S. Department of Energy \\ USDOE Office of Science (SC), \\ Basic Energy Sciences (BES) (SC-22)
}

Notice: This manuscript has been authored by employees of Brookhaven Science Associates, LLC under Contract No. DE-SC0012704 with the U.S. Department of Energy. The publisher by accepting the manuscript for publication acknowledges that the United States Government retains a non-exclusive, paid-up, irrevocable, world-wide license to publish or reproduce the published form of this manuscript, or allow others to do so, for United States Government purposes. 


\section{DISCLAIMER}

This report was prepared as an account of work sponsored by an agency of the United States Government. Neither the United States Government nor any agency thereof, nor any of their employees, nor any of their contractors, subcontractors, or their employees, makes any warranty, express or implied, or assumes any legal liability or responsibility for the accuracy, completeness, or any third party's use or the results of such use of any information, apparatus, product, or process disclosed, or represents that its use would not infringe privately owned rights. Reference herein to any specific commercial product, process, or service by trade name, trademark, manufacturer, or otherwise, does not necessarily constitute or imply its endorsement, recommendation, or favoring by the United States Government or any agency thereof or its contractors or subcontractors. The views and opinions of authors expressed herein do not necessarily state or reflect those of the United States Government or any agency thereof. 


\title{
Structural Evolution of Sub-10 nm Octahedral Platinum-Nickel Bimetallic Nanocrystals
}

Qiaowan Chang, ${ }^{\dagger}$ Yuan Xu, ${ }^{\dagger}$ Zhiyuan Duan, ${ }^{\dagger}$ Fei Xiao, ${ }^{\dagger}$ Fang Fu, ${ }^{\dagger}$ Youngmin Hong,${ }^{\S}$ Jeonghyeon Kim, ${ }^{\S}$ Sang-Il Choi, ${ }^{\S} *$ Dong Su, ${ }^{\ddagger} *$ Minhua Shao ${ }^{\dagger, \#, *}$

${ }^{\dagger}$ Department of Chemical and Biomolecular Engineering, Hong Kong University of Science and Technology, Clear Water Bay, Kowloon, Hong Kong, P.R. China

\$Center for Functional Nanomaterials, Brookhaven National Laboratory, Upton, New York 11973, United States

${ }^{\S}$ Department of Chemistry and Green-Nano Materials Research Center, Kyungpook National University, Daegu 41566, Korea

\# Energy Institute, Hong Kong University of Science and Technology, Clear Water Bay, Kowloon, Hong Kong, P.R. China

*E-mail: sichoi@knu.ac.kr,*E-mail: dsu@bnl.gov,*E-mail: kemshao@ust.hk Tel. $+852-34692269$

\begin{abstract}
Octahedral Pt alloy nanocrystals (NCs) have shown excellent activities as electrocatalysts toward oxygen reduction reaction (ORR). As the activity and stability of NCs are highly dependent on their structure and the elemental distribution, it is of great importance to understand the formation mechanism of octahedral NCs and to rationally synthesize shape-controlled alloy catalysts with optimized ORR activity and stability. However, the factors controlling the structural and compositional evolution during the synthesis have not been well understood yet. Here, we systematically investigated the structure and
\end{abstract}


composition evolution pathway of Pt-Ni octahedra synthesized with the assistance of $\mathrm{W}(\mathrm{CO})_{6}$ and revealed a unique core-shell structure consisting of a Pt core and a Pt-Ni alloy shell. Below $140{ }^{\circ} \mathrm{C}$, sphere-like pure Pt NCs with the diameter of 3-4 nm firstly nucleated, followed by the isotropic growth of Pt-Ni alloy on the seeds at temperatures between 170 and $230{ }^{\circ} \mathrm{C}$ forming Pt@Pt-Ni core-shell octahedra with $\{111\}$ facets. Owing to its unique structure, the Pt@Pt-Ni octahedra show an unparalleled stability during potential cycling, i.e., no activity drop after 10000 cycles between 0.6 and $1.0 \mathrm{~V}$. This work proposes the Pt@Pt-Ni octahedra as a high profile electrocatalyst for ORR and reveals the structural and composition evolution pathways of Pt-based bimetallic NCs.

Keywords: Pt-Ni octahedra; structural evolution; core-shell; oxygen reduction reaction; durability

TOC

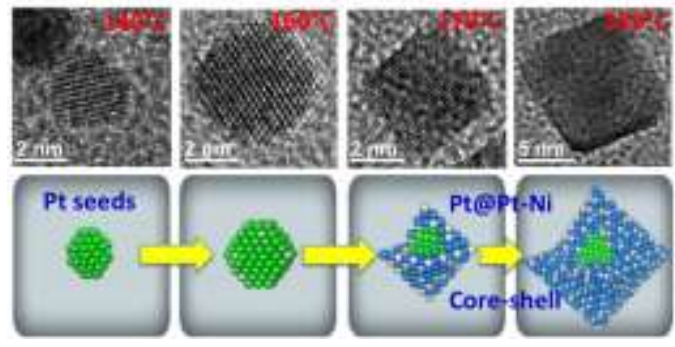


Oxygen reduction reaction (ORR) is the cathodic reaction for several important electrochemical systems such as fuel cells, metal-air batteries, corrosion, etc. ${ }^{1-8} \mathrm{Pt}$ nanocatalyst is believed as one of the best catalysts for ORR. However, its high price and the ever-increasing consumption from the industry largely impeded large-scale commercialization of fuel cells. To reduce the cost and boost the performance, significant progress has been made to optimize the multicomponent Pt-based electrocatalysts with desired size, composition and shape. ${ }^{1,4,8} \mathrm{Pt}$ alloying with transition metals (Pt-M) has shown higher ORR activity and stability than pure Pt due to the optimized oxygen binding energy. ${ }^{9-13}$ Particularly, their activities are highly dependent on the surface facets and it was found that octahedral Pt alloy nanocrystals (NCs) enriched with $\{111\}$ facets showed much higher ORR activity than conventional ones. ${ }^{1,4,11,14-23}$

There are two general approaches to synthesize octahedral bimetallic Pt-M NCs depending on whether CO-containing chemicals are used. ${ }^{14-16,21-24}$ Taking Pt-Ni as an example, in the synthesis protocol without involving CO-containing chemicals, $\mathrm{Pt}(\mathrm{acac})_{2}$ and $\mathrm{Ni}(\mathrm{acac})_{2}($ acac $=$ acetylacetonate $)$ mixed with appropriate solvents in a sealed autoclave were usually heated to a temperature between 120 and $200{ }^{\circ} \mathrm{C} .{ }^{16,22-23}$ The synthesized sub-10 nm octahedral Pt-Ni NCs showed an unusual composition distribution with Pt-rich frames and Ni-rich $\{111\}$ facets. ${ }^{24}$ Similar formation process was observed in the synthesis of $\mathrm{PtNi}_{3}$ rhombic dodecahedron, consisting of a Pt-rich frame with a Ni-rich interior phase. ${ }^{25}$ In the other synthesis approach including CO-containing chemicals, the mixture of $\mathrm{Pt}(\mathrm{acac})_{2}$ and $\mathrm{Ni}(\mathrm{acac})_{2}$, in the presence of $\mathrm{CO}$ or $\mathrm{W}(\mathrm{CO})_{6}$ via a degassing process was heated to $200-230{ }^{\circ} \mathrm{C} .{ }^{15,21}$ Although the importance of $\mathrm{W}(\mathrm{CO})_{6}$ and $\mathrm{CO}$ in controlling the morphology of Pt-Ni octahedra has been recognized by many reports, ${ }^{14-15,21}$ the structural and composition evolution pathways in this protocol have not been systematically investigated. Especially, the exact role of $\mathrm{W}(\mathrm{CO})_{6}$ in the morphology control of Pt-bimetallic NCs has not been demonstrated directly. 
In this work, for the first time, we systematically investigated the structure and composition evolution pathways of Pt-Ni octahedra synthesized with the assistance of $\mathrm{W}(\mathrm{CO})_{6}$ using various techniques, and established the correlation between ORR activities and their structures and compositions. A unique growth pathway for Pt bimetallic alloy NCs was discovered in this study. Small sphere-like Pt seeds initially formed at relatively low temperatures followed by the isotropic growth of Pt-Ni alloy on the seeds, resulting in an octahedral morphology. No phase segregation between $\mathrm{Pt}$ and $\mathrm{Ni}$ was observed during the growth of Pt-Ni layers, which is distinct from previous studies by Gan et al. ${ }^{24}$ and Niu et al. ${ }^{25}$ in their solvothermal synthesis without involving CO-containing species. The core-shell structure and uniform distribution of Pt and Ni atoms in the $\{111\}$ shells are responsible for the excellent stability of Pt-Ni octahedra during potential cycling.

Pt-Ni octahedra were prepared by following the previous report. ${ }^{15}$ The mixture of $\mathrm{Pt}(\mathrm{acac})_{2}, \mathrm{Ni}(\mathrm{acac})_{2}$, oleylamine $(\mathrm{OAm})$ and oleic acid (OAc) in benzyl ether was first purged with $\mathrm{Ar}$ and then $\mathrm{W}(\mathrm{CO})_{6}$ was added at $130{ }^{\circ} \mathrm{C}$ as the shape control agent. (see Supporting Information for details). In order to monitor the structural evolution with temperature, the intermediate samples at different temperatures $(140,160,170,180,190$, 210 , and $230{ }^{\circ} \mathrm{C}$ ) were taken out from the reactor and immediately cooled with toluene in an iced bath, and were then characterized by transmission electron microscopy (TEM), electron energy loss spectroscopy (EELS), X-ray diffraction (XRD), and X-ray photoelectron spectroscopy (XPS). 

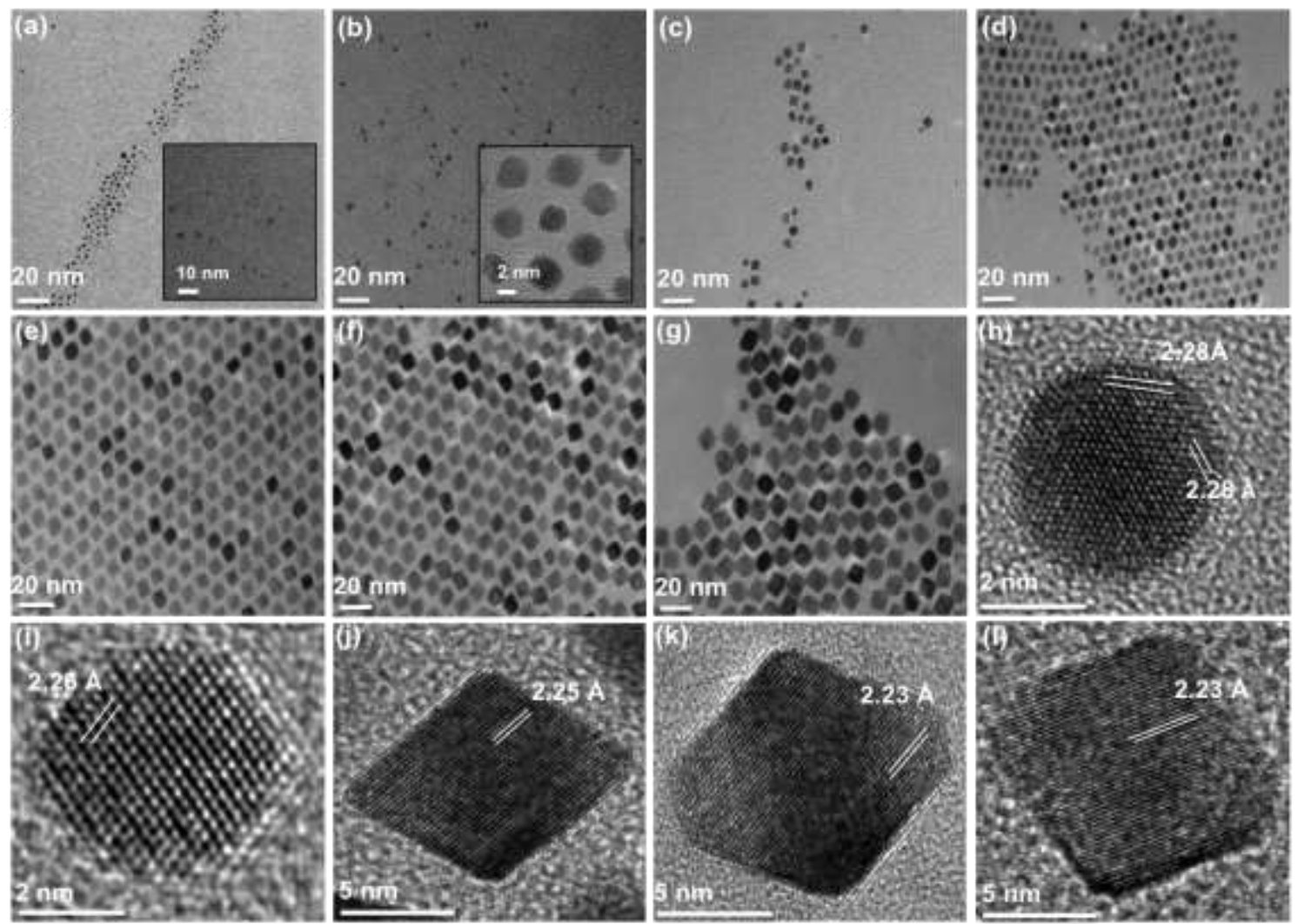

(m)

$\mathbf{i}$

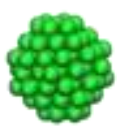

$140^{\circ} \mathrm{C}$ ii

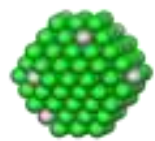

$160^{\circ} \mathrm{C}$

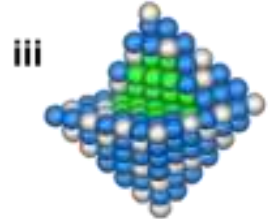

$170^{\circ} \mathrm{C}$

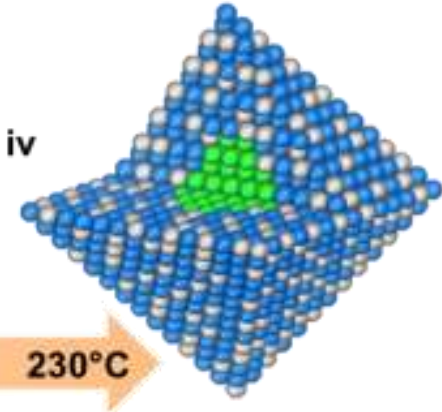

Figure 1. Low-magnification TEM images of as-obtained Pt-Ni nanocrystals at (a) $140{ }^{\circ} \mathrm{C}(3.0 \pm 1.0 \mathrm{~nm})$, (b) $160{ }^{\circ} \mathrm{C}(4.5 \pm 1.0 \mathrm{~nm})$, (c) $170{ }^{\circ} \mathrm{C}(5.4 \pm 0.3 \mathrm{~nm})$, (d) $180{ }^{\circ} \mathrm{C}$ $(5.7 \pm 0.3 \mathrm{~nm}),(\mathrm{e}) 190{ }^{\circ} \mathrm{C}(7.9 \pm 0.5 \mathrm{~nm})$, (f) $210{ }^{\circ} \mathrm{C}(9.0 \pm 0.5 \mathrm{~nm})$, and (g) $230{ }^{\circ} \mathrm{C}(9.5 \pm 0.5$ $\mathrm{nm}$ ). HRTEM images of invidual nanocrystal at (h) $140{ }^{\circ} \mathrm{C}$, (i) $160{ }^{\circ} \mathrm{C}$, (j) $180{ }^{\circ} \mathrm{C}$, (k) $210{ }^{\circ} \mathrm{C}$, and (1) $230{ }^{\circ} \mathrm{C}$. (m) A structural and composition evolution model for Pt-Ni octahedron. (i) Pure Pt sphere seeds with the diameter of 3-4 nm are formed at $140{ }^{\circ} \mathrm{C}$, (ii) then truncated octahedra with trace amount of $\mathrm{Ni}$ are formed at $160{ }^{\circ} \mathrm{C}$ with an average diameter of $4.5 \mathrm{~nm}$, (iii) then $5.4 \mathrm{~nm}$ octahedra at $170{ }^{\circ} \mathrm{C}$, and (iv) finally sub-10 nm 
octahedral Pt-Ni consisting of a Pt core and Pt-Ni shell are formed at $230{ }^{\circ} \mathrm{C}$. Pt atoms in the core: green; Pt atoms in the shell: blue; $\mathrm{Ni}$ atoms in the shell: grey.

Figure 1 shows the TEM images of Pt-Ni NCs obtained at various temperatures from $140-230{ }^{\circ} \mathrm{C}$. Before $\mathrm{W}(\mathrm{CO})_{6}$ was added, i.e., when temperature was lower than $130{ }^{\circ} \mathrm{C}$, no particle was formed. Sphere-like NCs were observed at $140^{\circ} \mathrm{C}$ (Figure 1a). Most of Pt NCs have a spherical shape with a diameter of $3.0 \pm 1.0 \mathrm{~nm}$, unlike a branched structure observed in the study of Gan et al. ${ }^{24}$ The lattice fringe of an individual NC in Figure $1 \mathrm{~h}$ is $\sim 2.28 \AA$, corresponding to that of the interplanar distance of (111) planes of pure Pt. This is also confirmed by the STEM-EELS line scan in Figure S1 where no Ni component was found. This result indicates that $\mathrm{Pt}(\mathrm{acac})_{2}$ was reduced first to form pure $\mathrm{Pt}$ seeds. The formation of pure Pt seeds at a relatively low temperature can be ascribed to the higher standard redox potential of $\mathrm{Pt}^{2+} / \mathrm{Pt}$ than that of $\mathrm{Ni}^{2+} / \mathrm{Ni}$, leading to the earlier reduction of Pt ions.

When temperature was further increased to $160{ }^{\circ} \mathrm{C}$ (Figure $1 \mathrm{~b}$ ), some sphere-like $\mathrm{Pt}$ seeds transformed into a truncated octahedral shape with an average diameter of $4.5 \pm 1.0$ nm. The truncated octahedral shape can be clearly seen in Figure 1i. The interplanar distance of truncated octahedra decreased to $2.26 \AA$, implying that $\mathrm{Ni}$ atoms can be reduced together with $\mathrm{Pt}$ at elevated temperature. When the temperature reached $170{ }^{\circ} \mathrm{C}$, nearly all NCs had an octahedral shape with an average edge length of $5.4 \pm 0.3 \mathrm{~nm}$ (Figure 1c). This result suggests that $170{ }^{\circ} \mathrm{C}$ is the transition point where truncated octahedra grow to octahedra.

As temperature further increases, Pt-Ni octahedra continued to grow. The average

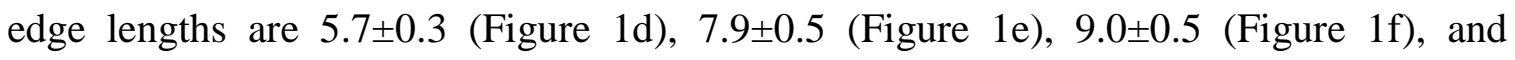
$9.5 \pm 0.5 \mathrm{~nm}$ (Figure 1g) at 180, 190, 210, and $230{ }^{\circ} \mathrm{C}$, respectively. The interplanar distances of Pt-Ni octahedra at 180, 210, and $230{ }^{\circ} \mathrm{C}$ are 2.25 (Figure 1j), 2.23 (Figure 
$1 \mathrm{k}$ ), and $2.23 \AA$ (Figure 11), respectively, which are typical values for the d-spacing of $\{111\}$ planes of Pt-Ni alloy. ${ }^{15}$ The number of atoms reduced at different temperature intervals can be estimated based on the size of the NCs and the atomic density of Pt-Ni $\left(\sim 74.7\right.$ atoms $\left.\mathrm{nm}^{-3}\right)$ with a Pt to Ni molar ratio of 1:1. Figure S2 clearly shows that most $\mathrm{Pt}$ and $\mathrm{Ni}$ ions were reduced between 180 and $210{ }^{\circ} \mathrm{C}$.

To gain insight into the elemental distribution of Pt-Ni NCs, the samples at 180 and $230{ }^{\circ} \mathrm{C}$ were characterized using aberration-corrected high-resolution STEM imaging and STEM-EELS. The HAADF-STEM images in Figure 2a-c clearly demonstrate the well-controlled octahedral morphology of Pt-Ni NCs at $180{ }^{\circ} \mathrm{C}$. Figure $2 \mathrm{~d}$ and e show the EELS-elemental mappings of Pt and Ni, respectively, for a single NC shown in Figure 2c. The well-resolved Ni signal in Figure 2e implies that Ni reduction occurs at temperature below $180{ }^{\circ} \mathrm{C}$. The Ni signal is much weaker in the center than other parts of the octahedron. In contrast, the Pt signal is stronger in the center (Figure 2d). The mappings of Pt and Ni suggest that the Pt-Ni octahedron consists of a Pt-rich and Ni-poor core, and a Pt-Ni shell with uniform distribution of Pt and Ni. The existence of the Pt-rich core is consistent with the formation of the pure Pt seeds at lower temperatures.

The HAADF-STEM images of the sample taken at $230{ }^{\circ} \mathrm{C}$ in Figure 3a-c, and $\mathrm{f}$ also demonstrate the well-controlled octahedral shape. EELS-elemental mappings for a single Pt-Ni octahedron shown in Figure $3 \mathrm{c}$ along the $\langle 100\rangle$ zone axis show clear Pt enrichment in the center compared to the shell (Figure 3d), while the Ni content in the center is much lower than other areas (Figure 3e). The elemental mapping results on another particle along the $\langle 110\rangle$ zone axis also show the same conclusion (Figure 3f-h). In the synthesis of Pt-Ni octahedra without involving CO-containing chemicals, Gan et $a l .^{24}$ found that a Pt-rich hexapod structure was formed firstly via a kinetically controlled process, followed by the deposition of a Ni-rich phase in the concave $\{111\}$ facets via a thermodynamically controlled step. In our work, hexapod Pt frame was not observed. 
Instead, a core-shell like structure consisting of a pure Pt core and Pt-Ni alloy shell was formed.
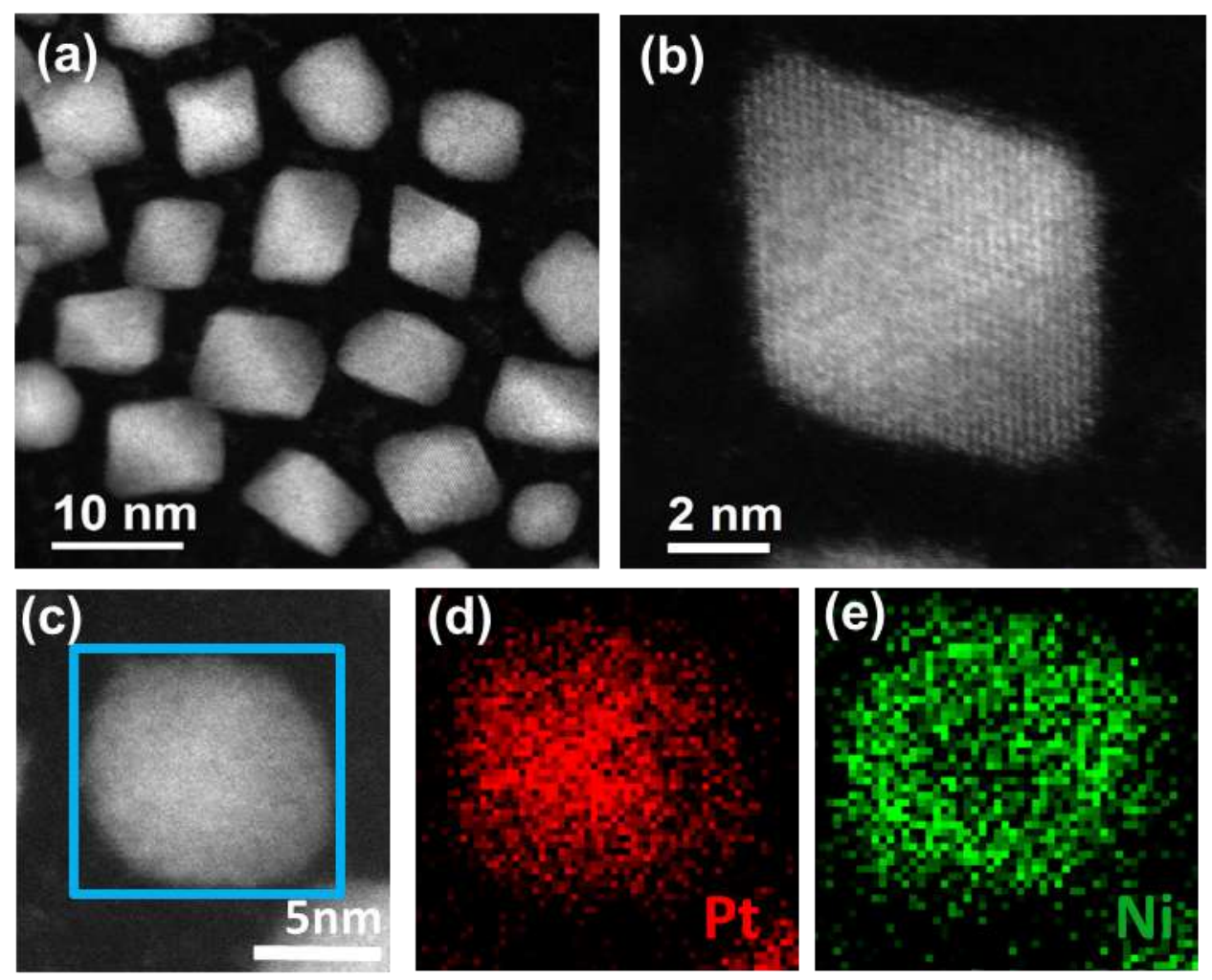

Figure 2. (a) ADF-STEM image of Pt-Ni octahedra at $180{ }^{\circ} \mathrm{C}$. (b) Atomic resolution HAADF-STEM image from a single Pt-Ni octahedron along the <110> zone axis. (c) ADF-STEM image and (d, e) EELS element maps of a single Pt-Ni octahedron present the distribution of $\mathrm{Pt}$ (red) and $\mathrm{Ni}$ (green). 

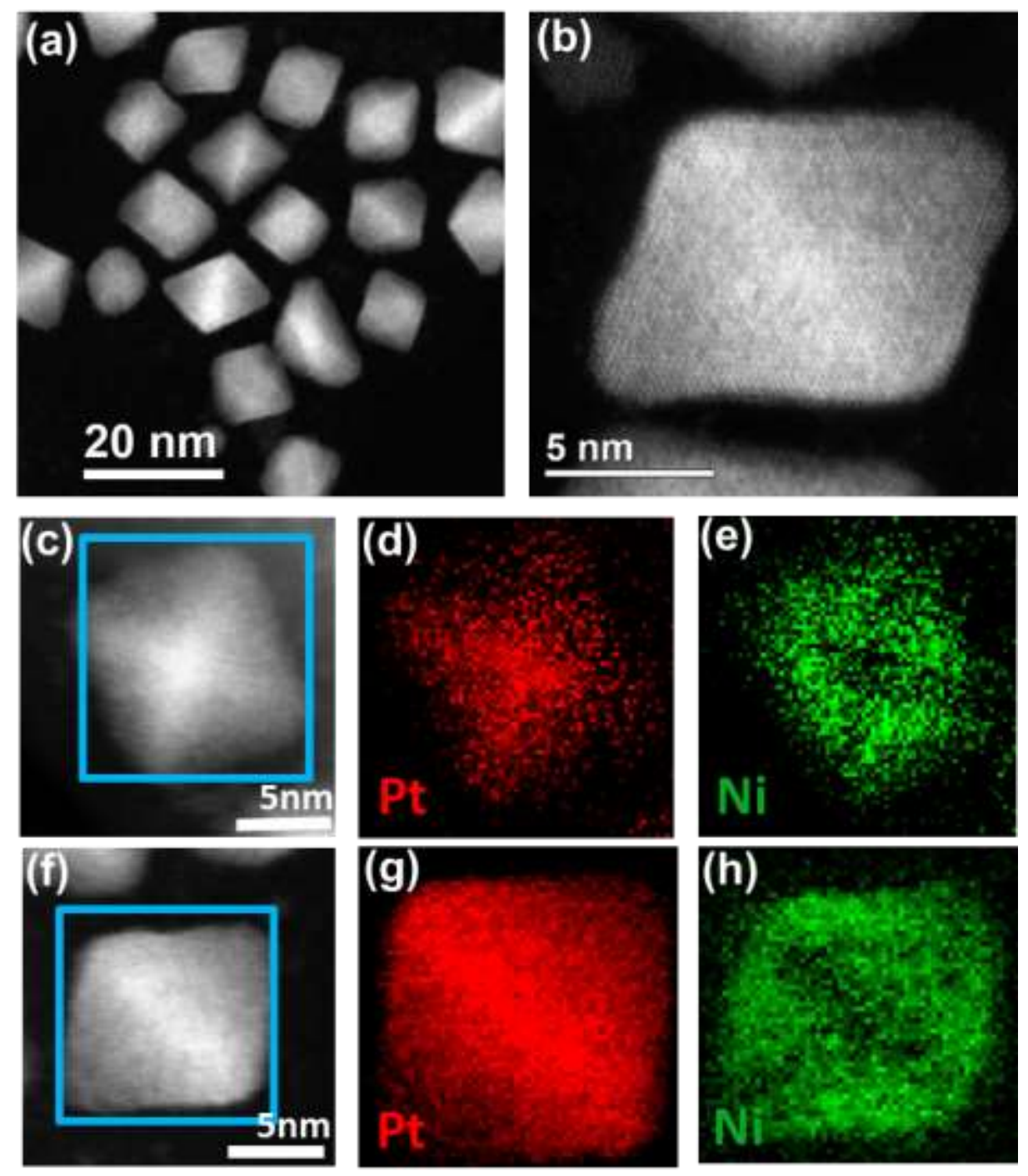

Figure 3. (a) ADF-STEM image of Pt-Ni octahedra at $230{ }^{\circ} \mathrm{C}$. (b) Atomic resolution HAADF-STEM image from a single Pt-Ni octahedron. ADF-STEM images and STEM-EELS element maps of Pt and Ni from a single Pt-Ni octahedron recorded along the $(\mathrm{c}-\mathrm{e})<100>$ zone axis and $(\mathrm{f}-\mathrm{h})<110>$ zone axis.

The structure and composition of Pt-Ni NCs were examined by ICP-MS, XPS, and XRD. Table S1 lists the compositions of samples at different temperatures derived from ICP-MS and XPS results. The contents of $\mathrm{Ni}$ in samples at temperatures below $170{ }^{\circ} \mathrm{C}$ 
are too small to have an accurate calculation. A general trend observed in Table S1 is that the Pt to Ni ratio decreases as temperature increases, regardless of the measurement methods. This trend is consistent with the assumption that pure Pt seeds form first, which leads to higher $\mathrm{Pt}$ to $\mathrm{Ni}$ ratios at lower temperatures. Furthermore, the Pt-Ni layers deposited at a higher temperature should have a lower Pt to Ni ratio since Ni atoms could be easily reduced at high temperatures. The XRD patterns in Figure S3 also support this argument. At $160{ }^{\circ} \mathrm{C}$, the positions of XRD peaks are very close to those of pure $\mathrm{Pt}$, consistent with other characterization results. With increasing reaction temperature, the peaks gradually shift to higher $2 \theta$ angles, suggesting that more Ni atoms exist in NCs. The other observation in Table S1 is that the Pt to Ni ratios estimated from the ICP-MS are always larger than that from XPS at the same temperature. This discrepancy is due to a short maximum sampling depth (1 to $5 \mathrm{~nm}$ ) of the X-ray beam in XPS. It is possible that some of the Pt atoms in the core were not sampled resulting in a smaller Pt to $\mathrm{Ni}$ ratio than that obtained by ICP-MS.

Our X-ray fluorescence (XRF) result reveals the existence of a small amount of W $\left(\sim 2.4\right.$ atom\%) in the Pt-Ni sample at $230{ }^{\circ} \mathrm{C}$ (Figure S4a). When the reactor was not sealed well, the percentage of $\mathrm{W}$ in the product increased to $18.0 \%$ (Figure S4b), implying that the amount of $\mathrm{W}$ residues increases with a higher oxygen level in the reaction solution. The $\mathrm{W} 4 \mathrm{f}$ doublet with the binding energies of 35.4 and $37.6 \mathrm{eV}$ in Figure $\mathrm{S} 5$ can be assigned to $\mathrm{W}^{6+}$ species. To understand the oxidation state change of $\mathrm{W}$ at different temperatures, an experiment without adding $\mathrm{Pt}$ and $\mathrm{Ni}$ precursors was conducted. The solution was colorless until the temperature reached at $210{ }^{\circ} \mathrm{C}$, at which it changed to dark blue due to the formation $\mathrm{WO}_{2.9} \cdot{ }^{26-27}$ The color further changed to yellow at $230{ }^{\circ} \mathrm{C}$ due to the formation of $\mathrm{WO}_{3.0}{ }^{28-29}$ These results imply that $\mathrm{W}$ formed via the decomposition of $\mathrm{W}(\mathrm{CO})_{6}$ can be oxidized to $\mathrm{W}$ oxides by trace of $\mathrm{O}_{2}$ in the reaction solution at $210{ }^{\circ} \mathrm{C}$ and higher. 
To understand the role of $\mathrm{W}$ and $\mathrm{CO}$ in reducing $\mathrm{Pt}$ and $\mathrm{Ni}$ precursors, the experiment using $\mathrm{CO}$ instead of $\mathrm{W}(\mathrm{CO})_{6}$ was conducted. As shown in Figure S6, the color of the reaction changed from yellow $\left(140{ }^{\circ} \mathrm{C}\right)$ to brown $\left(160{ }^{\circ} \mathrm{C}\right)$ and black $\left(180{ }^{\circ} \mathrm{C}\right)$ with increasing temperature when $\mathrm{W}(\mathrm{CO})_{6}$ was applied. When $\mathrm{CO}$ was used, the solution color changed in the same sequence but at a much slower rate from 140 to $180{ }^{\circ} \mathrm{C}$. This result suggests that $\mathrm{W}$ may play a key role in promoting the reduction of $\mathrm{Pt}(\mathrm{acac})_{2}$ and $\mathrm{Ni}(\mathrm{acac})_{2}$. Based on the above observations, the possible role of $\mathrm{W}(\mathrm{CO})_{6}$ can be proposed as below. $\mathrm{W}(\mathrm{CO})_{6}$ firstly decomposes to generate metallic $\mathrm{W}$, which reduces $\mathrm{Pt}(\mathrm{acac})_{2}$ and $\mathrm{Ni}(\mathrm{acac})_{2}$ and forms $\mathrm{W}^{x+}$ ions. In the presence of trace of oxygen, $\mathrm{W}^{x+}$ ions are oxidized to $\mathrm{WO}_{2.9}$ and then to $\mathrm{WO}_{3.0}$. Our results provide a direct evidence confirming the hypothesis that $\mathrm{W}$ could accelerate the reduction of $\mathrm{Pt}$ and $\mathrm{Ni}$ ions proposed by Zhang et $a l .{ }^{21}$ and Niu et al. ${ }^{30}$

In order to examine the loaction of $\mathrm{W}$ in the product, EELS-elemental mappings for multiple particles formed at $230{ }^{\circ} \mathrm{C}$ were conducted and shown in Figure S7. Besides octahedral Pt-Ni particles, irregular $\mathrm{WO}_{x}$ nanoparticles were also found. To clarify whether $\mathrm{W}$ doping in the Pt-Ni particles occurred during the synthesis, EELS-elemental scan for a Pt-Ni octahedron was conducted and shown in Figure S8. It is clear that W atoms mainly located in the shell (surface or near the surface) of the Pt@PtNi core-shell particle. In the Pt core, the $\mathrm{W}$ content was negligible. This result confirms the incorporation of $\mathrm{W}$ in the product during the $\mathrm{W}(\mathrm{CO})_{6}$-assisted synthesis of Pt-Ni octahedra And $\mathrm{W}$ incorporation mainly occurs together with the formation of the Pt-Ni shell.

Based on above experimental results and discussions, a general pathway of Pt-Ni octahedra formation is proposed and shown in Figure $1 \mathrm{~m}$. At temperatures below $140{ }^{\circ} \mathrm{C}$, $\mathrm{Pt}(\mathrm{acac})_{2}$ were reduced with the assistance of $\mathrm{W}$ forming $\sim 3 \mathrm{~nm}$ sphere-like pure Pt seeds, which began to transform into truncated octahedra at $\sim 160{ }^{\circ} \mathrm{C}$. At higher temperatures, $\mathrm{Pt}$ and $\mathrm{Ni}$ ions were co-reduced. With the assistance of $\mathrm{CO}$ generated from the 
decomposition of $\mathrm{W}(\mathrm{CO})_{6}$, Pt and $\mathrm{Ni}$ atoms might mainly deposit on (100) surfaces resulting in an octahedral shape. The morphology transformation to octahedron was nearly completed at $170{ }^{\circ} \mathrm{C}$. Most of the Pt and Ni ions, however, were reduced between 180 and $210{ }^{\circ} \mathrm{C}$ with the edge length increasing from 5.7 to $9.0 \mathrm{~nm}$. In this step, Pt and $\mathrm{Ni}$ atoms were deposited uniformly on the surface following an epitaxial growth mechanism, resulting in a core-shell structure consisting of a Pt-rich core and a uniform Pt-Ni shell. This mechanism is also in good agreement with our previous work on the deposition of an ultrathin $(\sim 1 \mathrm{~nm}$ in thickness $)$ Pt-Ni shell on Pd NCs in a two-pot synthesis. ${ }^{31}$ It is worth noting that $\mathrm{W}(\mathrm{CO})_{6}$ not only provides $\mathrm{CO}$ to control the morphology of $\mathrm{Pt}-\mathrm{Ni}$, but also generates $\mathrm{W}^{0}$ to facilitate the reduction of $\mathrm{Pt}$ and $\mathrm{Ni}$ ions. The exact role of $\mathrm{W}(\mathrm{CO})_{6}$ in controlling the morphology requires more detailed study.

We further correlate the structure and composition with ORR activity of samples formed at different temperatures. For $\mathrm{Pt}-\mathrm{Ni} \mathrm{NCs}$ formed at $170{ }^{\circ} \mathrm{C}$ and lower temperatures, the surfactants (OAm/OAc) could not be removed easily. Figure S9 compares the XPS spectra of $\mathrm{N} 1 \mathrm{~S}$ of representative samples at 170,180 , and $230{ }^{\circ} \mathrm{C}$. A strong N 1s signal $(\sim 398 \mathrm{eV})$ could be seen in the $170{ }^{\circ} \mathrm{C}$ sample, indicating the existence of residual surfactants on the surface. In contrast, it was negligible in other two samples. The high coverage of surfactants on the surface for the sample formed at $170{ }^{\circ} \mathrm{C}$ is also reflected in the cyclic voltammogram (CV, Figure S10). The CVs of samples at 180 and $230{ }^{\circ} \mathrm{C}$ show typical features of Pt alloys, ${ }^{32}$ while the faradic currents associated with $\mathrm{H}$ adsorption/desorption and oxide formation/reduction for the sample at $170{ }^{\circ} \mathrm{C}$ are much smaller due to surface contaminations. At low temperature, the surface is mainly composed of $\mathrm{Pt}$ atoms. With increasing temperature, the $\mathrm{Ni}$ percentage gradually increases on the surface of NCs, resulting in a lower coverage of surfactants. Based on this finding, we conclude that the interaction between surfactants and surface of Pt-Ni 
NCs may be dependent on the surface composition: a stronger interaction is expected to exist on a surface with a higher Pt to Ni ratio.

The oxygen reduction polarization curves of Pt-Ni/C samples at 170, 180, 190, 210 and $230{ }^{\circ} \mathrm{C}$ in oxygen-saturated $0.1 \mathrm{M} \mathrm{HClO}_{4}$ solutions are shown in Figure 4a. For comparison, the curve for a commercial Pt/C (TKK, 46\%) is also included. It is clear that the ORR activity of the sample at $170{ }^{\circ} \mathrm{C}$ is very low due to surface contamination. The polarization curves of other samples are parallel to that of $\mathrm{Pt} / \mathrm{C}$ in the mixed and kinetic controlled regions, and have a very similar limiting current density suggesting negligible surface contamination. It is worth noting that Pt loadings on rotating disk electrodes (RDEs) varies for different samples. Kinetic currents are normalized to the Pt mass on the $\mathrm{RDE}$ and real surface area derived from CO stripping, and are compared in Figure $4 \mathrm{~b}$ and c, respectively. The Pt mass activities at $0.9 \mathrm{~V}$ (vs RHE) of Pt-Ni/C samples at 180, 190, 210 , and $230{ }^{\circ} \mathrm{C}$ are $0.64,1.58,1.75$, and $2.00 \mathrm{~A} \mathrm{mg}^{-1}$, respectively (Table S2). These values are also much higher than that of $\mathrm{Pt} / \mathrm{C}\left(0.20 \mathrm{~A} \mathrm{mg}^{-1}\right)$. Even though the average particle size increases with increasing temperature resulting in a lower Pt utilization, the Pt mass activity increases as a function of temperature. This trend is mainly caused by the optimization of $\mathrm{Pt}$ to Ni ratio during the synthesis resulting in higher specific activities. Figure $4 \mathrm{c}$ and Table S2 clearly show that the specific activity also increases with temperature due to larger particle sizes and optimized Pt to Ni ratios.
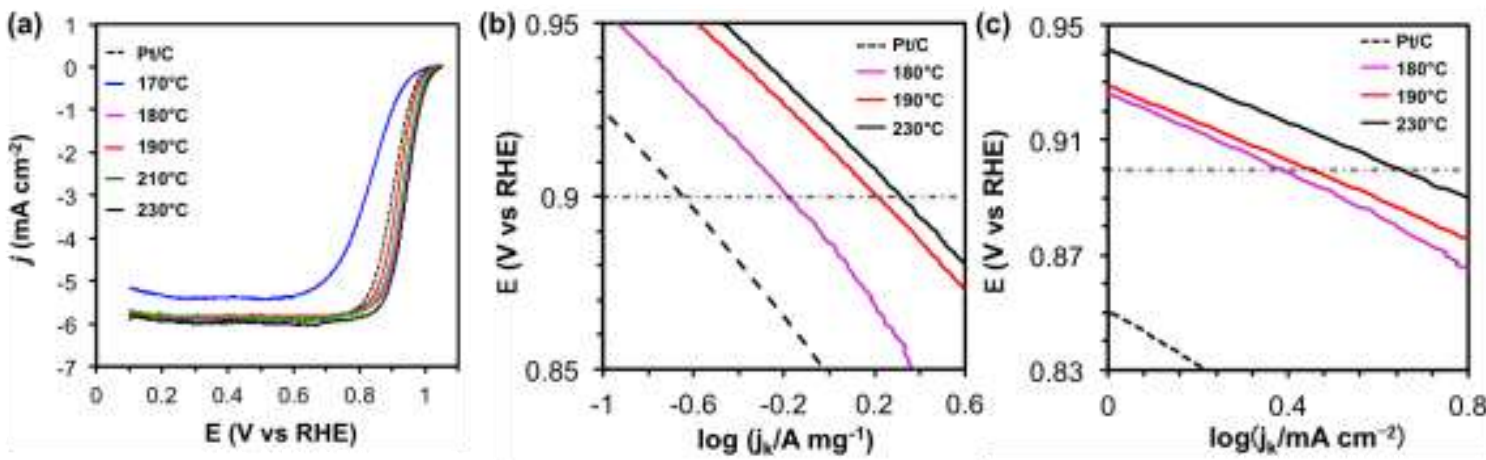
Figure 4. (a) The positive-going ORR polarization curves of Pt-Ni/C samples at 170, 180, 190, 210, and $230{ }^{\circ} \mathrm{C}$, and a commercial Pt/C (TKK) in an $\mathrm{O}_{2}$-saturated $0.1 \mathrm{M} \mathrm{HClO}_{4}$ solution with a scanning rate of $10 \mathrm{mV} \mathrm{s}^{-1}$ and the rotation speed of $1600 \mathrm{rpm}$. Pt loadings on the RDE are 5.00, 6.80, 1.48, 1.86, 2.94, and 5.00 $\mu$ g for Pt-Ni/C samples at 170, 180, 190, 210, and $230{ }^{\circ} \mathrm{C}$ and $\mathrm{Pt} / \mathrm{C}$, respectively. The Tafel plots of (b) mass activity and (c) specific activity derived from (a).

Potential cycling was conducted in order to gain more insights into the structure of Pt-Ni octahedra. A triangle cycling protocol was adapted by cycling the $\mathrm{Pt}_{2.4} \mathrm{Ni} / \mathrm{C}$ formed at $230{ }^{\circ} \mathrm{C}$ between 0.60 and $1.0 \mathrm{~V}$ with a scanning rate of $50 \mathrm{mV} \mathrm{s}^{-1}$ in an $\mathrm{O}_{2}$-saturated $0.1 \mathrm{M} \mathrm{HClO}_{4}$ solution. The $\mathrm{CVs}$ and oxygen reduction polarization curves before and after 5000 and 10000 cycles are compared in Figure 5a and 5b, respectively. The as-synthesized sample showed a couple of small current peaks in the region of 0.35 and $0.7 \mathrm{~V}$, possibly associated with $\mathrm{Ni}-\mathrm{OH}$ species. These currents disappeared after cycling implying that $\mathrm{Ni}$ atoms in the surface were removed. There is no noticeable change in ORR polarization curves with only a couple of $\mathrm{mV}$ shift in the half-wave potential before and after potential cycling in Figure 5b. The distributions of Pt and Ni, however, change significantly. As shown in Figure 5c, the octahedral shape was maintained after 10000 cycles. The Ni atoms were removed from the surface and sublayers of Pt-Ni octahedra, resulting in an octahedral nanostructure consisting of a Pt-rich shell, a Pt-Ni alloy interlayer and a Pt-rich core (Figure 5f). The excellent stability of Pt-Ni octahedra under the current testing condition can be ascribed to the formation of a thick (1-1.5 nm) and stable Pt layer. ${ }^{33,34}$ This structure and composition evolution pathways found in this study are also drastically different from that observed by Gan et al. ${ }^{24}$ In their case, a pure Pt nanopod was formed during potential cycling due to dissolution of $\mathrm{Ni}$ in the $\mathrm{Ni}$-rich facets. Based on these results, we conclude that the structural and composition evolution 
mechanisms in both formation and degradation processes of Pt-bimetallic octahedra are highly dependent on the synthesis methods.
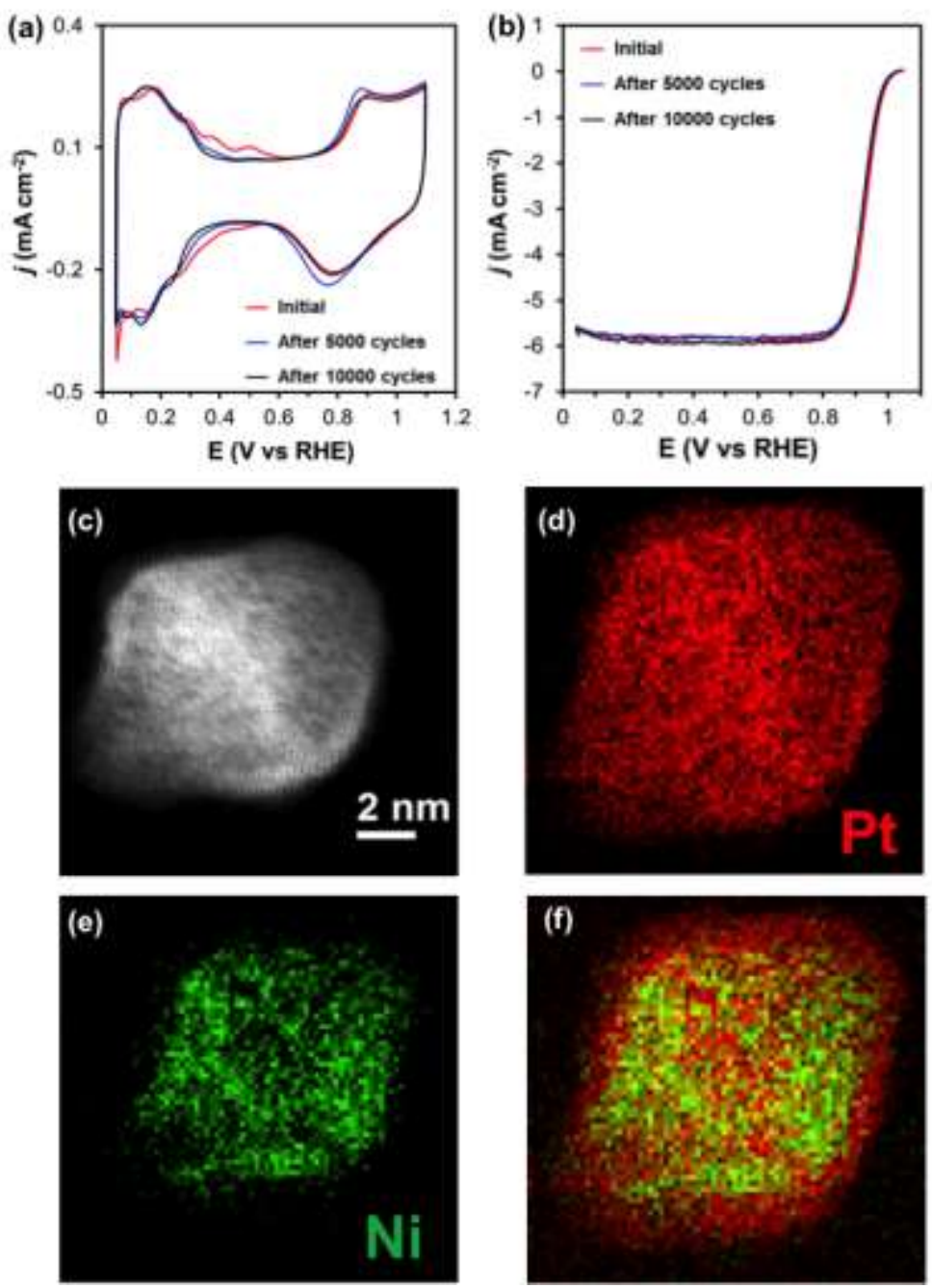

Figure 5. (a) $\mathrm{CVs}$ in a $\mathrm{N}_{2}$-saturated $0.1 \mathrm{M} \mathrm{HClO}_{4}$ solution and (b) ORR polarization curves in an $\mathrm{O}_{2}$-saturated $0.1 \mathrm{M} \mathrm{HClO}_{4}$ solution of octahedral $\mathrm{Pt}_{2.4} \mathrm{Ni} / \mathrm{C}$ synthesized at $230{ }^{\circ} \mathrm{C}$ before (red line), and after 5000 (blue line) and 10000 (black line) cycles. The Pt loading is $10.2 \mu \mathrm{g} \mathrm{cm}^{-2}$. (c) ADF-STEM image of a single Pt-Ni octahedron after 10000 potential cycles. (d-f) The corresponding STEM-EELS element maps: Pt (d, red) and Ni (e, green) and their mixture (f). 
In conclusion, a systematic study on formation pathways of Pt-Ni octahedra in a synthesis protocol involving $\mathrm{W}(\mathrm{CO})_{6}$ was conducted. Intermediate Pt-Ni NCs obtained at various temperatures were characterized by HRTEM, EELS, XRD, XPS and RDE. Our results revealed a unique structural and composition evolution pathway of Pt-Ni octahedra. Pure spherical Pt seeds with the diameter of 3-4 nm were formed at $140{ }^{\circ} \mathrm{C}$ and gradually changed to a truncated octahedron at $160{ }^{\circ} \mathrm{C}$. The morphology transformation to octahedron was nearly completed at $170{ }^{\circ} \mathrm{C}$. Most of $\mathrm{Pt}$ and $\mathrm{Ni}$ atoms were reduced between 180 and $210^{\circ} \mathrm{C}$. This step involved the epitaxial growth of Pt-Ni layers, resulting in a core-shell structure consisting of a Pt-rich core and a uniform Pt-Ni shell. Moreover, our results also provide a direct evidence confirming that $\mathrm{W}(\mathrm{CO})_{6}$ not

only provides $\mathrm{CO}$ to control the morphology of $\mathrm{Pt}-\mathrm{Ni}$, but also generates $\mathrm{W}^{0}$ to accelerate the reduction of $\mathrm{Pt}$ and $\mathrm{Ni}$ ions. The unique core-shell structure and uniform distribution of $\mathrm{Pt}$ and $\mathrm{Ni}$ in the $\{111\}$ shell is responsible for the high ORR activity and stability. Our results also imply that it is possible to replace the Pt core with other cheaper materials under this unique structural and composition evolution mechanism. These insights will provide a guideline to rational synthesis of shape-controlled bimetallic electrocatalysts with desired electrocatalytic activity and stability. Further studies on the structural and composition changes of bimetallic NCs under different electrochemical and thermal tests are highly recommended.

\section{ASSOCIATED CONTENT}

\section{Supporting Information}

Experimental details, atom numbers and color variation figures of Pt-Ni samples, ADF-STEM images and elemental mappings, XRD of Pt-Ni samples, XRF spectra, XPS 
spectra of $\mathrm{W}$ and $\mathrm{N}$, and $\mathrm{CVs}$, ORR activities of $\mathrm{Pt}-\mathrm{Ni} / \mathrm{C}$ samples,. This material is available free of charge via the Internet at http://pubs.acs.org.

\section{AUTHOR INFORMATION}

\section{Corresponding Authors}

*Email: sichoi@knu.ac.kr; dsu@bnl.gov; kemshao@ust.hk

\section{Notes}

The authors declare no competing financial interest.

\section{ACKNOWLEDGMENT}

The authors acknowledge the support from Research Grant Council (IGN13EG05 and 26206115), Innovation and Technology Fund (ITS/323/14) of the Hong Kong Special Administrative Region, and a startup fund from the Hong Kong University of Science and Technology. The electron microscopy work was carried out at the Center for Functional Nanomaterials, Brookhaven National Laboratory, which is supported by the U.S. Department of Energy (DOE), Office of Basic Energy Sciences, under Contract No. DE-SC-00112704. The work at Kyungpook National University was supported by the Basic Science Research Program through the National Research Foundation of Korea (NRF) funded by the Ministry of Education, Science and Technology (NRF-2015R1D1A3A01019467).

\section{References}

1. Shao, M.; Chang, Q.; Dodelet, J.-P.; Chenitz, R., Chem. Rev. 2016, 116, 3594-3657.

2. Luntz, A. C.; McCloskey, B. D., Chem. Rev. 2014, 114, 11721-11750.

3. Stratmann, M.; Müller, J., Corros. Sci. 1994, 36, 327-359.

4. Markovic, N.; Schmidt, T.; Stamenkovic, V.; Ross, P., Fuel Cells. 2001, 1, 105-116.

5. Laoire, C. O.; Mukerjee, S.; Abraham, K.; Plichta, E. J.; Hendrickson, M. A., J. Phys. 
Chem. C 2009, 113, 20127-20134.

6. Wang, Z.-L.; Xu, D.; Xu, J.-J.; Zhang, X.-B., Chem. Soc. Rev. 2014, 43, 7746-7786.

7. Cheng, F.; Chen, J., Chem. Soc. Rev. 2012, 41, 2172-2192.

8. Nie, Y.; Li, L.; Wei, Z., Chem. Soc. Rev. 2015, 44, 2168-2201.

9. Stamenkovic, V. R.; Mun, B. S.; Arenz, M.; Mayrhofer, K. J.; Lucas, C. A.; Wang, G.;

Ross, P. N.; Markovic, N. M., Nat. Mater. 2007, 6 , 241-247.

10. Hwang, S. J.; Kim, S.-K.; Lee, J.-G.; Lee, S.-C.; Jang, J. H.; Kim, P.; Lim, T.-H.; Sung, Y.-E.; Yoo, S. J., J. Am. Chem. Soc. 2012, 134 , 19508-19511.

11. Choi, S. I.; Xie, S.; Shao, M.; Lu, N.; Guerrero, S.; Odell, J. H.; Park, J.; Wang, J.; Kim, M. J.; Xia, Y., ChemSusChem 2014, 7 , 1476-1483.

12. Gong, K.; Chen, W.-F.; Sasaki, K.; Su, D.; Vukmirovic, M. B.; Zhou, W.; Izzo, E. L.; Perez-Acosta, C.; Hirunsit, P.; Balbuena, P. B., J. Electroanal. Chem. 2010, 649, 232-237. 13. Bian, T.; Zhang, H.; Jiang, Y.; Jin, C.; Wu, J.; Yang, H.; Yang, D., Nano Lett. 2015, $15,7808-7815$.

14. Wu, J.; Gross, A.; Yang, H., Nano Lett. 2011, 11, 798-802.

15. Choi, S.-I.; Xie, S.; Shao, M.; Odell, J. H.; Lu, N.; Peng, H.-C.; Protsailo, L.; Guerrero, S.; Park, J.; Xia, X., et al., Nano Lett. 2013, 13, 3420-3425.

16. Cui, C.; Gan, L.; Li, H.-H.; Yu, S.-H.; Heggen, M.; Strasser, P., Nano Lett. 2012, 12, 5885-5889.

17. Huang, X.; Zhao, Z.; Cao, L.; Chen, Y.; Zhu, E.; Lin, Z.; Li, M.; Yan, A.; Zettl, A.; Wang, Y. M., et al., Science 2015, 348, 1230-1234.

18. Park, J.; Zhang, L.; Choi, S.-I.; Roling, L. T.; Lu, N.; Herron, J. A.; Xie, S.; Wang, J.; Kim, M. J.; Mavrikakis, M., et al., ACS Nano 2015, 9, 2635-2647.

19. Zhao, X.; Chen, S.; Fang, Z.; Ding, J.; Sang, W.; Wang, Y.; Zhao, J.; Peng, Z.; Zeng, J., J. Am. Chem. Soc. 2015, 137, 2804-2807.

20. Park, J.; Liu, J.; Peng, H. C.; Figueroa - Cosme, L.; Miao, S.; Choi, S. I.; Bao, S.; Yang, X.; Xia, Y., ChemSusChem 2016, 9, 2209-2215.

21. Zhang, J.; Yang, H.; Fang, J.; Zou, S., Nano Lett. 2010, 10, 638-644.

22. Wu, Y.; Cai, S.; Wang, D.; He, W.; Li, Y., J. Am. Chem. Soc. 2012, 134, 8975-8981.

23. Carpenter, M. K.; Moylan, T. E.; Kukreja, R. S.; Atwan, M. H.; Tessema, M. M., J. Am. Chem. Soc. 2012, 134, 8535-8542.

24. Gan, L.; Cui, C.; Heggen, M.; Dionigi, F.; Rudi, S.; Strasser, P., Science 2014, 346, 1502-1506.

25. Niu, Z.; Becknell, N.; Yu, Y.; Kim, D.; Chen, C.; Kornienko, N.; Somorjai, G. A.; Yang, P., Nat. Mater. 2016, 15, 1188-1194

26. Li, Y. H.; Liu, P. F.; Pan, L. F.; Wang, H. F.; Yang, Z. Z.; Zheng, L. R.; Hu, P.; Zhao, H. J.; Gu, L.; Yang, H. G., Nat. Commun. 2015, 6, 8064-8070.

27. Rothschild, A.; Frey, G.; Homyonfer, M.; Tenne, R.; Rappaport, M., Mater. Res. Innovations 1999, 3, 145-149. 
28. Takahashi, T.; Iwahara, H., J. Appl. Electrochem. 1973, 3, 65-72.

29. Turkez, H.; Cakmak, B.; Celik, K. Key Eng. Mater., 2013, 543, 89-92.

30. Niu, G.; Zhou, M.; Yang, X.; Park, J.; Lu, N.; Wang, J.; Kim, M. J.; Wang, L.; Xia, Y., Nano Lett. 2016, 16, 3850-3857.

31. Choi, S.-I.; Shao, M.; Lu, N.; Ruditskiy, A.; Peng, H.-C.; Park, J.; Guerrero, S.; Wang, J.; Kim, M. J.; Xia, Y., ACS Nano 2014, 8, 10363-10371.

32. Shao, M.; Odell, J. H.; Choi, S.-I.; Xia, Y., Electrochem. Commun. 2013, 31, 46-48.

33. Wang, D.; Xin, H. L.; Hovden, R.; Wang, H.; Yu, Y.; Muller, D. A.; DiSalvo, F. J.; Abruña, H. D., Nat. Mater. 2013, 12, 81-87.

34. Pan, Y.T., Wu, J., Yin, X. and Yang, H., AIChE Journal, 2016, 62(2), 399-407. 